\title{
LAUT- UND AKZENTGESCHICHTLICHES ZU EINIGEN AUS SUBSTRATEN UND NACHBARSPRACHEN INS SLAVISCHE ENTLEHNTEN NAMEN
}

In diesem Beitrag ${ }^{1}$ werden einige Topo-, Hydro-, Choro- und Ethnonyme, die das Slavische aus Sprachen entlehnt hat, die im Zuge seiner Expansion zu seinen Substraten oder Nachbarn geworden waren, auf ihre laut- und akzentgeschichtlichen Besonderheiten hin untersucht. Die hier ausgewählten Namen stammen aus dem Griechischen, Lateinischen bzw. Romanischen, „Alteuropäischen“, Thrakischen und anderen indogermanischen Substratsprachen, dem Deutschen und anderen germanischen Dialekten, dem Finnischen und dem Iranischen. Soweit möglich und sinnvoll, wird hier das in Holzer 2011b präsentierte Modell der slavischen und gegebenenfalls romanischen Lautgeschichte zugrunde gelegt; dieses ist zwar auf das neuštokavische Kroatische gemünzt, gilt aber über weite Strecken auch für andere slavische Sprachen und Dialekte. Die in lautgeschichtliche Herleitungen eingefügten kleinen Ziffern verweisen auf die Paragraphen in Holzer (2011b), in denen die betreffenden Lautgesetze behandelt werden: römische Ziffern auf romanische und arabische Ziffern auf slavische Lautgesetze. Bei aufrecht gedruckten arabischen Ziffern handelt es sich um gewöhnliche Lautgesetze, bei kursiv gedruckten hingegen um Lautsubstitutionen. ${ }^{2}$

Nota bene: Die Akzentstelle wird durch Unterstreichung ( _ ) angegeben (steht an der betreffenden Stelle ein infralineares Diakritikon, weicht die Unterstreichung um eine Stelle nach links aus). Ein der Silbe nachgestellter Punkt bezeichnet, wenn er ganz oben steht ('), den alten Akut, und wenn er auf halber Höhe steht (·), den Neoakut. Das drucktechnisch schwierige $\check{e}$ mit Makron wird hier durch $\hat{e}$ ersetzt; somit steht $\hat{e}$ für langes und ě für kurzes Jat.

\section{Solkan}

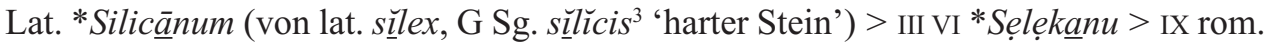
* Selẹkānu (friaul. Salcan und ital. Salcano sind aus dem Slovenischen übernommen)

* Institut für Slawistik der Universität Wien, Spitalgasse 2, Hof 3, A-1090 Wien, Österreich; georg.holzer@univie.ac.at

1 Er geht auf meinen Vortrag „Die ältesten Entlehnungen fremder Orts- und Flussnamen ins Slavische“" (gehalten am 13. Juni 2015 auf dem XXX. Namenkundlichen Symposium in Kals am Großglockner, 11.-13. Juni 2015) und das zugehörige ausführliche Handout zurück.

2 Zu Lautsubstitutionen s. Holzer (1996a: 69 § 3.4.3).

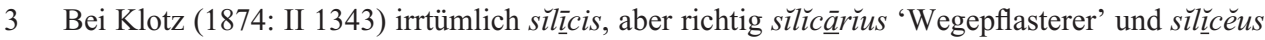
'aus Kieselstein'. Vgl. die loc. cit. aus Ovid, Metamorphosen IX angeführten Hexameter 225 und 304 sowie Georges (1862: 1508): G Sg. sĭlǐcis. 
$>78$ nachurslav. *Silikāa $n u$ (entlehnt nach der Dritten Palatalisierung (1)) > sloven. Solkän, Gen. Solkána. Vgl. Šekli (2009: 122), Snoj (2009: 388-389). Hier wurde im Zuge der Entlehnung der „Entl e h n ung s-A k u t“ eingefügt (8). Eine Regel, die vorhersagbar machen würde, unter welchen Umständen eine fremde lange betonte Silbe von den Slaven als akutiert interpretiert und daher bei der Entlehnung ein Akut eingefügt wurde und unter welchen nicht, konnte bislang nicht gefunden werden. ${ }^{4}$ Der von Saskia Pronk-Tiethoff aus germanischen Lehnwörtern abstrahierten Regel (s. Pronk-Tiethoff 2013: 264-273) widersprechen überaus zahlreiche Entlehnungen aus dem Romanischen (s. Holzer 2011b: 36-38) und auch manche der hier behandelten Namen. Der Eindruck der Willkürlichkeit wird durch das Vorhandensein von Dubletten (s. Holzer 2011b: 38) verstärkt.

Solkan liegt links der Soča (ital. Isonzo $0^{5}$ ) bei Nova Gorica und östlich der Isoglosse, die das Westromanische mit Sonorisierung zwischen Vokalen oder nach Vokal, aber vor $r$ stehender stimmloser Konsonanten vom Ostromanischen ohne Sonorisierung trennt. $^{6}$

\section{Sužid}

Lat. *Silicêtum (ebenfalls von lat. sillex, s. o.) > III VI *Sẹlẹkẹtu > IX und mit westromanischer Sonorisierung $k>g, t>d$ und mit ,italienischer" Palatalisierung $g>d \check{z}$ rom. *Selẹdžę $d u>78$ ursl. *Silidžl $d u$ (mit Entlehnungs-Akut) > sloven. *Solžìd, Gen. *Solžída (standardisiert zu Sužíd). Vgl. Šekli (2009: 122), Snoj (2009: 402).

Sužid liegt westlich von Karfreit/Kobarid (s. u.) und auf der westlichen Seite der Sonorisierungs-Isoglosse (s. Šekli 2009: 116). Abgesehen von der verschiedenen Suffigierung stellt sloven. Solkän : *Solžïd ein ebensolches ostromanisch-westromanisches Entsprechungspaar dar wie lat. cìvitạtem > kroat. Càvtat: Čabdad und lat. aurā $\underline{t} t a$ (als Fischname) > kroat. òvrata : orâda (s. Holzer 2011b: 98, 99-100, 137).

\section{Kobarid}

Lat. *Caprêttum (von caper, G Sg. capri 'Bock' oder capra 'Geiß’) > III VI *Kaprẹtu > IX und mit westromanischer Sonorisierung und eingefügtem $o$ rom. ${ }^{*} K a b o \underline{r e} e d u$ (1184 in villa, que dicitur Kauoretum iuxta Isuncium) $>78$ ursl. *Kabarī du (mit

4 Siehe Holzer (2005: 60), Šekli (2009: 126). Meine in (Holzer 2009: 153) angestellten Überlegungen zum Fehlen einer solchen Regel (,lautstilistische oder individuelle Varianten“ in der germanischen bzw. romanischen Aussprache betonter Silben, zum Beispiel mit und ohne „creakyvoice") hat Pronk-Tiethoff (2013: 270-271) ohne wirkliche Begründung abgelehnt.

5 Deutsch 1401 an die Ysnicz, ca. 1440 an der Snicz (s. Snoj 2009: 386), Isenz (s. Kranzmayer 1956: 43 Fußnote 17).

6 Siehe Šekli (2009: 115, 116), aber auch Kranzmayer (1956: 42-43 Fußnote 17), der den Unterschied zwischen den ins Slavische übernommenen Namen mit Sonorisierung und denen ohne Sonorisierung nicht geographisch, sondern chronologisch erklärt, und zwar dahingehend, dass erstere vor diesem westromanischen Lautwandel und letztere danach ins Slavische entlehnt worden wären. Seine chronologische Erklärung einer offenbar geographischen Distribution zwingt Kranzmayer zu siedlungsgeschichtlichen Ad-Hoc-Hypothesen. 
Entlehnungs-Akut) > sloven. Koborìd, Gen. Koborída (standardisiert zu Kobarìd; friaul. *Kaborẹedu > Cjaurêt, ital. Caporetto, dt. *Kafrît > Karfreit mit volksetymologischer Einwirkung von Karfreitag). Vgl. Ramovš (1921-1922: 60-62), Šekli (2009: 121), Snoj (2009: 191).

Schwierigkeiten macht nicht nur die Einfügung des $o$ im Romanischen, ${ }^{7}$ sondern auch die Tatsache, dass dieses $o$ im Slavischen durch $a$ und nicht durch $u(>25 b)$ substituiert wurde (7). Vielleicht handelt es sich um eine Assimilation an die erste Silbe.

\section{Solun}

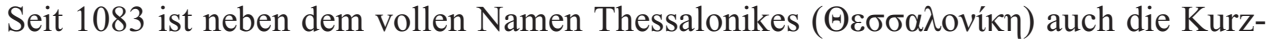
form $\dot{\eta} \Sigma \alpha \lambda$ ovík bezeugt, die aber erheblich älter sein muss, weil sie über romanische Vermittlung schon vor dem an den Anfang des 9. Jahrhunderts zu datierenden Wandel $a>o$ ins Slavische gelangt ist (s. Schramm 1981: 342). ${ }^{8}$ Die Stadt heißt auf Altbulgarisch Solunъ, auf Russisch Солунь (Gen. Солуни, fem.) und auf Neuštokavisch Sòlūn, Gen. Solúna (s. Vasmer 1979: 693, Holzer 2011b: 150). Das slovenische Solún, Gen. Solúna (s. Snoj 2009: 535) stammt nach Marko Snojs brieflicher Auskunft „etwa vom Ende des 19. Jh. Im 16. Jh. liest man bei Dalmatin in der Bibelübersetzung S. Pavla Lyst na Tessaloniharje."

Zwar war das $i$ in víkn 'Sieg' ursprünglich lang, aber „,[s]pätestens zu Ende der Antike waren die Quantitätsoppositionen allgemein aufgehoben" und alle Vokale kurz (s. Rix 1976: $57 \S 65,41 \S 47) .{ }^{9}$ Mit bereits kurzem $i$ wurde $\Sigma \alpha \lambda$ ovíkๆ ins Lateinische übernommen und dort gemäß der lateinischen „Pänultimaregel“ mit Betonung auf der drittletzten Silbe *Salonica ausgesprochen. (Damit gehört diese Entlehnung der Kategorie Q an, das sind diejenigen Entlehnungen, in denen sich die lateinische Betonung - phonologisch irrelevant, wie die Betonung im Lateinischen ja war - unabhängig von der griechischen ausschließlich nach der phonologisch relevanten Quantität der Pänultima richtete. ${ }^{10}$ ) Dann aber ist es zu zwei volksetymologischen Missverständnissen gekommen. Auf das eine, nämlich dass in das -ic- $(<$ gr. -ík-!) das der Ableitung von Adjektiven dienende lateinische Suffix ic- hineininterpretiert worden ist, hat bereits Gottfried Schramm hingewiesen. Allerdings geht Schramm hinsichtlich der Quantitäten fehl, wenn er schreibt: „*Salona ist eine plausible lat. Verkürzung von *Salōnica, denn diese Lautung ließ sich als fem. Adjektiv auffassen, zu dem civitas hinzugedacht wurde." (Schramm 1981:343). ${ }^{11}$ Von gr. $\Sigma \alpha \lambda$ ovíkๆ kommt man nicht zu lat. „*Salōnica“, sondern nur zu *Salonica mit kurzem $o$. Und von diesem *Salonica konnte nur ein * Salona rückgebildet worden sein, mit Initialbetonung

7 Erklärungsversuche (Anaptyxe oder Analogie) finden sich in Ramovš (1921-1922: 62).

8 Zur Datierung des Wandels s. Holzer (2011b: 58).

9 Vgl. auch Karvounis (2008: 67-69), ferner Holzer (2011a: 81, mit Fußnote 1) und zu diesem Toponym 84.

10 Siehe Holzer (2011a: 84); dort ist *Salona auf * Salona zu korrigieren.

11 Zur selben „Verkürzung“ kam es in lat. Tarsatica $>$ kr. Tr̀̀sāt, vgl. Schramm (1981: 343) und Holzer (2014: 206). 
nach der Pänultimaregel (vgl. angẹlicus : angelus). Das zweite volksetymologische Missverständnis bestand darin, dass dieses * Salona als eines der vielen Toponyme auf $\underline{\text { on }} a^{12}$ interpretiert wurde (vgl. Verona, Ancona, Cremona usw.), was eine Anpassung von *Salona an diesen Ortsnamentyp nach sich zog. So hat *Salona eine lange und daher auch betonte Pänultima bekommen. Erst nach diesem volksetymologischen Eingriff hatte der Name die Lautung *Salōna, die Schramm ohne Erklärung ansetzt. Dieses *Salōna wandelte sich dann lautgesetzlich zu romanischem *Salōna und wurde so ins Urslavische (mit $\bar{g}$ als Fremdphonem) übernommen, wo es wie viele andere feminine Städtenamen in die maskuline o-Deklination übergeführt wurde (vgl. Skok 1921-22: 25) und somit *Salōnnu lautete. ${ }^{13}$ Dieses ergab dann nach dem Wirken des Dyboschen Gesetzes (11), dem Wandel $a>o$ (23), dem Vollzug des Zweiten Ivšićschen Gesetzes (37) und weiterem Lautwandel im Altbulgarischen Solunb und im Neuštokavischen Sòlūn. Das zu den i-Stämmen übergewechselte russische Солунь stimmt im Nominativ und im Akkusativ prosodisch mit der neuštokavischen Lautung überein, die anderen Kasus, die eigentlich endbetont sein sollten, haben sich an den Nominativ und den Akkusativ angeglichen.

Die Slaven erlangten spätestens gegen Ende des 6. Jahrhunderts Kenntnis von der Stadt und ihrem Namen, nämlich als sie 586 oder 597 die Stadt belagerten. Mit dem Einlass slavischer (urslavischer!) Überläufer in die Stadt war der Grundstein für die Entwicklung des slavischen Idioms gelegt, das 863 von Konstantin dem Philosophen zum „Urkirchenslavischen“, wie Trubetzkoy es nannte, verschriftet wurde. (Siehe Holzer 2006.)

\section{Solin}

Für das aus vorrömischem Substrat ${ }^{14}$ stammende lateinische Salōna, den Namen der Hauptstadt der römischen Provinz Dalmatien, ist durch Lukan in De bello civili IV

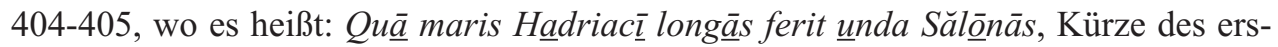
ten $a$ und Länge des $o$ verbürgt. Beides geht auch daraus hervor, dass der Name nach seiner Übernahme ins Slavische dort *Salūnu (ohne Entlehnungs-Akut) lautete, was über *Solȳn $\underline{\underline{z}}$ das heutige kroatische Sòlīn (so in neuštokavischer Akzentuierung) ergab. ${ }^{15}$ Auch diese Entlehnung erfolgte offensichtlich vor dem Wirken des Dyboschen Gesetzes (11), dem Wandel $a>o$ (23) und dem Vollzug des Zweiten Ivšićschen Gesetzes (37). Völlig zufällig kommen sowohl in der Vorgeschichte des slavischen Namens Thessalonikis als auch in der des slavischen Namens Salonas die Lautungen lat. (*) Salōna > VI IX rom. **Salọnna vor. Der lautliche Unterschied zwischen kr. Sòlūn und kr. Sòlin ergibt sich alleine daraus, dass der Name Salonas erst nach dem romanischen Wandel XII $\bar{o}>\bar{u}$ (rom. *Salōna $>$ rom. *Salūna) ins Slavische übernommen wurde und dieses $\bar{u}$ daher den Wandel des urslavischen $\bar{u}$ zu nachursl. $\bar{y}$ (15) mitgemacht hat, der Name Thessalonikes jedoch schon vor dem romanischen Wandel $\bar{o}>\bar{u}$, also noch mit

$12 \mathrm{Zu}$ diesem Zugehörigkeitssuffix s. Anreiter (2001: 76 Fußnote 293).

13 Zum Lautgeschichtlichen siehe Holzer (2011b: 150).

14 Vgl. zuletzt Anreiter (2001: 20).

15 Zum Lautgeschichtlichen siehe Holzer (2011b: 149). 
$\bar{o}$ ins Slavische entlehnt wurde, das dann den Anschluss an die Weiterentwicklung des aus dem urslavischen Diphthong aw entstandenen heimischen $\bar{o}(3,17)$ zu nachursl. $\bar{u}$ (17) fand (vgl. Holzer 2011b: 150 s. v. Sòlūn).

Soweit ich sehe, fehlt auch allen anderen Substratnamen auf -ōna der EntlehnungsAkut. Jedenfalls gilt dies für die kroatischen Toponyme Skràdīn, Làbìn, Jàkīn, Nìn, Nòrīn (s. Holzer 2011b: 92 ff. s. vv.) und Plömin ${ }^{16}$ sowie für die slovenischen Ortsnamen (lat. *Bergōna $>$ VI *Bẹrgọna $>\mathrm{IX} *$ Bẹrgọna $>$ XII rom. *Bẹrgūna $>7$ ursl. *Birgūnn >) Bargín, Gen. Bargína (standardisiert zu Bregínj) und (lat. *Glemōna [ca. 610 in Glemona] > VI *Glẹmona > IX *Glẹ̣ōna > XII rom. *Glemūna $>7$ ursl. *Glimūnu >) *Golmín, Gen. *Golmina (standardisiert zu Gumín) (vgl. Šekli 2009: 121, Snoj 2009: 77-78, 156). Vielleicht haben die Slaven in allen eventuell vorhanden gewesenen Varianten dieses Ausgangs richtig ein und dasselbe „Ortsnamensuffix“ erkannt und im auch bei Volksetymologien zum Tragen kommenden Bedürfnis nach lautlicher Vereinheitlichung vermeintlich oder tatsächlich etymologisch identischer Morpheme die nichtakutierte Variante dieses Suffixes verallgemeinert. Das Umgekehrte könnte mit den im Lateinischen auf das Ortsnamensuffix - $\underline{\underline{e}} t u m$ ausgehenden Namen geschehen sein, vgl. ursl. *Silidž̄ ${ }^{*} d u$ und *Kabarī $d u$ (s. o.) sowie lat. bruscềtum (von brusscum 'Auswuchs, Knorren') > kroat. Bruškït. ${ }^{17}$ Wo das Ortsnamensuffix -êtum nicht als solches erkannt wurde, etwa weil es in eine weitere Ableitung eingebaut war, konnte es ohne Akut entlehnt werden; ein Beispiel scheint das deminutive lat. *Cannêtulus (von canna 'Rohr') > čak. Kanîtal auf $\operatorname{Rab}^{18}$ zu sein.

Die Slaven erlangten spätestens um 600 Kenntnis von Salona und seinem Namen, als sie vor der Stadt erschienen und Papst Gregor der Große dies in seinem Brief an Salonas Bischof Maximus erwähnte (siehe Holzer 2011b: 1 Fußnote 3.).

\section{Srědbcb}

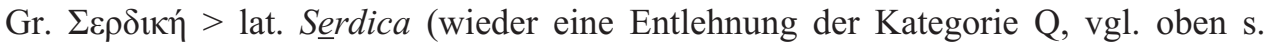
v. Solun) > vI rom. *Serdẹka $>78$ ursl. *Ser ${ }^{*}$ diku (mit Entlehnungs-Akut und wieder mit Überführung in die maskuline o-Deklination $\left.{ }^{19}\right)>121620242543$ mittelbulg. Srě ${ }^{b c c b}$ 'Sofia'. ${ }^{20}$ Entlehnt vor dem ältesten nachurslavischen Lautgesetz, der Dritten Palatalisierung, und somit eine urslavische Entlehnung (vgl. Holzer 2008: $75 \S 1.5$.).

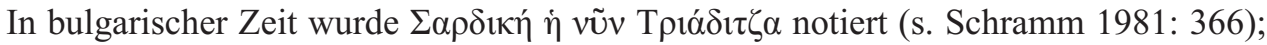
diese Akzentuierung und der hier erfolgte Wandel $\underline{\underline{e}}>j \underline{a}$ sprechen für Initialbetonung

16 Lat. Flanōna > kroat. Plömin wurde, wie es scheint, erst nach dem Wirken des Dyboschen Gesetzes (neu)entlehnt und dekliniert daher ohne Akut nach dem Akzentparadigma a (s. Holzer 2011b: 140).

17 Lagename auf Rab. Siehe Skok (1950: 58, 59) und Holzer (2014: 208), mit weiteren Literaturangaben. Mit dalmatischem $k$ und unsonorisiertem $t$, entlehnt nach der Zweiten Palatalisierung (7) und entweder auch nach dem Wandel $u>b$ (25), also nicht vor der ersten Hälfte des 9. Jh., oder aber mit unregelmäßiger romanischer Dehnung in der ersten Silbe, vgl. Holzer (2014: 201-203 § (I)).

18 Siehe Skok (1972: 29) und die Erläuterungen in Holzer (2014: 203 Fußnote 11).

19 Nach Schramm (1981: 365) wurde in Anlehnung an das Genus von grad b aus dem L Sg. Srě dbci ein Maskulinum abstrahiert.

20 Einzelheiten zu diesem Namen s. in Schramm (1981: 364-366). 
und die Tatsache, dass Dybos Gesetz nicht gewirkt hat, für Akut in der ersten Silbe. Auch die Wiedergabe des lateinischen $e$ durch rom. $e$ (nicht $e)^{21}>$ slav. $e$ (nicht $i$; s. Holzer 2011b: 35 § 7) spricht für Initialbetonung.

Serdica lag nördlich der sog. Jireček-Skok-Linie, die eine Latein sprechende nördliche Hälfte der Balkanhalbinsel von einer Griechisch sprechenden südlichen trennte; ${ }^{22}$ denn: „Die spätantiken Inschriften aus Serdica sind in ihrer Mehrheit lat. abgefaßt“; wohl ist es so, dass „im 5.-6. Jh. der Vorrang vom Griech. auf das Lat. überging“, womit „unwahrscheinlich ist, daß die Slawen in Serdica auf ein griech. Übergewicht trafen" (s. Schramm 1981: 365).

\section{Timok}

Aus dem Thrakischen: etwa $* t ə m-a k^{w} \bar{a}$ 'schwarzes Wasser' ${ }^{23}>$ lat. Timacus (Plinius, Naturalis historia III 149) > III VI *Temaku > IX rom. *Tẹmaku $>7$ ursl. *Tèmaku (ohne Entlehnungs-Akut) $>11 *$ Tima $\underline{k} u>2325 *$ Timmoks $>$ (mit dialektaler Kürzung? ${ }^{24}$ ) *Timokr $>3948$ *Timok $>69$ serb. Tìmok, Gen. Timoka. Mündet, von Serbien kommend, im Dreiländereck zwischen Serbien, Rumänien und Bulgarien in die Donau.

Meine in Holzer (2014: 202) geäußerte Annahme ${ }^{25}$ einer romanischen prosodischen Sonderentwicklung gebe ich jetzt auf und gehe von der in Pravopis 1960: 735 angegebenen Akzentuierung Timok, Gen. Tìmoka und von der in Ristić/Kangrga (1928: 1054) und Skok (1973: 470) angegebenen Akzentuierung Tìmočanin (ursl. *Tịmačjā ne) und tìmočk $\bar{l}$ aus. Das lateinische $a$ muss angesichts seines nachurslavischen Reflexes $o$ kurz und nach der Pänultimaregel daher unbetont gewesen sein.

„A. 818 stellte sich eine Gesandtschaft von Slawen, die vom Timok [...] nach Heristal an der Maas angereist war, Ludwig dem Frommen als Timociani $[\ldots]$ ( $=*$ Timočane) vor." (Schramm 1981: 146.)

\section{Sutomore}

Zum lateinischen Maria geben Klotz (1874: I 361) und Georges (1862: 210) Măaria und Mărīa als Akzentuierungsvarianten an. Der Name ist wohl aus dem Griechischen entlehnt, wo er Mapía lautet. Lat. Mărīa ist eine Entlehnung der Kategorie A, also

21 Nur betontes lateinisches $e$ bleibt im Romanischen offenes $e$; siehe Holzer (2011b: 23-24 $\S \mathrm{VI}$ ).

$22 \mathrm{Zu}$ dieser Linie siehe Holzer (2011b: 10-11).

23 Vgl. Popović (1960: 76, 98, 154, 172; nach Mladenov und Georgiev). Zur Bedeutung von 'weiß' und 'schwarz' in Flussnamen s. Holzer (2008: 9-30).

24 Eine Akzentuierung Tìmok, Gen. Tìmoka kann es nach standardserbischen Lautgesetzen nur als Ergebnis einer Akzentverschiebung von der ersten Silbe auf die zweite nach Dybos Gesetz und danach der neuštokavischen Akzentverschiebung geben. Das aber schließt den Akut aus, den man nach standardserbischen Lautgesetzen der Kürze des $i$ wegen in der ersten Silbe ansetzen müsste. In ARj XVIII 33 sind mehrere Belege des Hydronyms aus dem Volkslied zitiert, dessen Überregionalität eine dialektale Zuordnung der Akzentuierung erschwert.

25 Sie baut auf die in ARj XVIII 331 und in Skok (1973: 470) angegebene Akzentuierung Tìmok, Gen. Timòka auf. Skok (1973: 470) merkt an: „U rimsko doba Timacus (s akcentom na -a)“ — woher weiß er das? - Zum Namen Timok siehe noch Holzer (2008: 76, 107, 201 Fußnote 6). 
mit Längung der akzentuierten Pänultima zur Beibehaltung der Akzentstelle, Mă ăra hingegen ist eine Entlehnung der Kategorie Q, also mit Beibehaltung der Quantitäten und Betonung nach der lateinischen Pänultimaregel (s. Holzer 2011a: 85-86). ${ }^{26}$ Beide Akzentuierungen scheinen in slavischen Entlehnungen vorzukommen, und zwar Mă $\breve{r} \breve{a}$ in Sütomore und Mărīa in Sutomôrje (s. u.). Zunächst zu Sütomore:

Lat. Sancta Maria > II IV *Sanç̧ta Márja > VII *Sanç̧ta Már'a > 36 (8) ursl. *San (')xta Marja. Mit Entlehnungs-Akut oder ohne. Die wahrscheinlich nach $600 \mathrm{n}$. Chr. zu datierende romanische Längung IX hat hier nicht stattgefunden, was diese Entlehnung aus dem Romanischen als eine der ältesten ausweist (s. Holzer 2011b: 26).

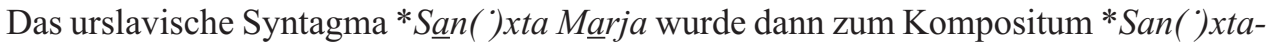
marja univerbiert. Zur urslavischen Betonungslosigkeit solcher Komposita vgl. ursl. *čirnagal 'wu> nšt. crìnoglāv 'Schwarzkopf', *lej( ')stapā 'du> nšt. lïstopād 'Oktober' u. v. a. Die weitere Entwicklung verlief lautgesetzlich: Ursl. *San( ')xtamarja $>2$ (12) 142328 *Sōtomorje > 303344 * Sotomor'e > 4761 montenegr. Sütomore. Siehe ARj XVII 80, Skok (1973: 365), Šimunović (1986: 112, 118); Holzer (1998: 29), Holzer (2011b: 153 s. v. sutal.).

\section{Sutomorje}

Lat. Sancta Marīa > II rom. *Sançta Marīa $>6$ (8) ursl. *San ( )xta Marija. ${ }^{27}$ Dieses urslavische Syntagma wurde dann zum Kompositum *San( ')xtamarija univerbiert. Zur Akzentuierung solcher Komposita vgl. ursl. *čirna wal suju 'der, dessen Haar schwarz ist', ursl. *zal 'tagal 'wu 'einer, dessen Kopf golden ist' u. v. a. Die weitere Entwicklung verlief wieder lautgesetzlich: Ursl. *San(')xtamarija > 211 (12) 14232528 *Sōtomorbje $>414748 *$ Sutomorje > 67 čak. oder altštok. (also ohne neuštokavische Akzentverschiebung 69) Sutomôrje (Äcker). Der Name scheint in Skok (1973: 365 auf; ohne Lokalisierung).

\section{Logatec}

Lat. Longaticum > III VI IX rom. *Longāatẹku $>7$ ursl. *Lungāatiku (ohne EntlehnungsAkut) > sloven. Logátec (dt. Loitsch). Eine sehr frühe Übernahme vor der Dritten Palatalisierung. Vgl. Holzer (1996b: 85), Holzer (2008: 75 § 1.5), Snoj (2009: 243). Zur ostromanischen Herkunft des Namens ohne Sonorisierung siehe Šekli (2009: 116).

$26 \mathrm{Zu}$ sieben anderen solchen A/Q-Dubletten siehe Holzer (2011a: 86).

27 Wohl wurde das lateinische antevokalische $\underline{i}$ im Urslavischen zu $i j$ zerlegt (vgl. ursl. ${ }^{*} k r \bar{u}{ }^{*}$ : *kruwe). 


\section{Oglej}

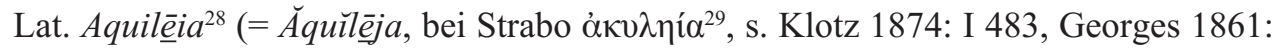

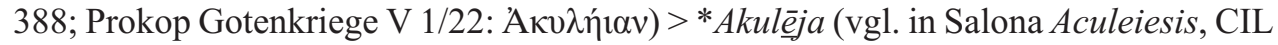
12925, s. Katičić 1980: 29) > VI *Akuleja > IX und mit westromanischer Sonorisierung rom. *Agulēja ${ }^{30}$ (friaul. Aghulẹa > Aguléa; s. Skok 1921-22: 31, Kranzmayer 1956: 43 Fußnote 17, Katičić 1980: 29) > 8 ursl. *Agulē ju (mit Entlehnungs-Akut) $>2 * A g u l \underline{e} \dddot{j} i$

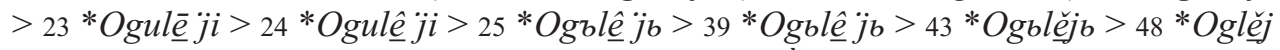
$>$ ekavisch (statt 60) $*$ Oglej $>67 *$ Oglẹj $>69$ kroat. $\dagger \grave{O g l e j j ~(s o ~ h y p o t h e t i s c h ~ a k z e n t u i e r t ~}$ in HER 862; vgl. Oglej in der Istrischen Grenzziehung), ikavisch Oglij (Kožičić), sloven. Oglêj, mhd. Agley, Aglay. Siehe ARj VIII 748, Skok (1921-22: 26), Skok (1953: 52-53), Skok (1972: 547-548), Katičić (1980: 29), Anreiter (2001: 145), Holzer (2008: 140), Snoj (2009: 528).

Warum hier lat. $j$ ohne Assibilierung (> VIII $d z$ ) geblieben ist, diskutiert Skok (192122: 25-29, 32, 151). Vom Romanisten Paul Videsott (Bozen) wurde mir freundlich mitgeteilt, dass im Friaulischen wie auch im ehemals angrenzenden Dolomitenladinischen intervokalische $j, d j$ und $g j$ durch $j$ vertreten sind, und Peter Anreiter (Innsbruck) hat mir den Erhalt des $j$ anhand folgender Beispiele erörtert: lat. *Matrêia $>$ dt. Matrei (Tabula Peutingeriana: Matreio für Matrei am Brenner; urk. 1155-1164 Matrei für Matrei in Osttirol), friaul. Modréja (am Isonzo; s. auch Kranzmayer 1956: 42 Fußnote 17); lat. *Stupe(i)a > dt. Stubai (ca. 1000 hoba inter Alpes Stupeia, 11. Jh. mons in Stubey: linkes Seitental des Tiroler Silltales) und lat. Aquilệia > dt. Aglei. In diesem Zusammenhang hat Anreiter auch auf 893 Oneia, 1237 Unay im Pustertal und auf lat. Celêia > sloven. Celje hingewiesen. Siehe dazu auch Anreiter (1997: 83 Fußnote 557), Anreiter (2001: 145-146). Zu klass. lat. Celêiia > ,sloven. *Cel'é (morda bolje *Celbjë)“" siehe noch Snoj (2009: 87).

\section{Videm}

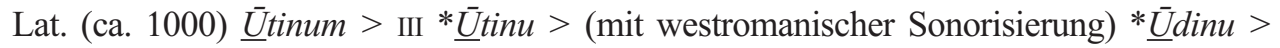
VI * $\underline{U} d e ̣ n u>$ IX rom. * $\underline{U} d e ̣ n u$ (friaul. Udin, vgl. ital. Udine) $>7$ ursl. * $\underline{U}$ dinu (ohne Ent-

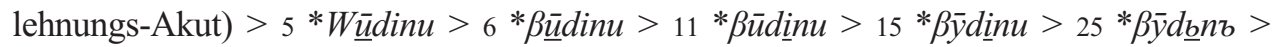

28 Zur Länge des $\underline{\bar{e}}$ s. Skok (1921-22: 26).

29 Entlehntes lateinisches $q u$ ergibt im Griechischen $\kappa$ mit Labialisierung des nachfolgenden Vokals

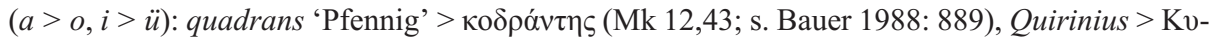

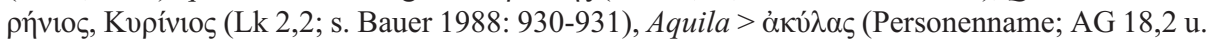

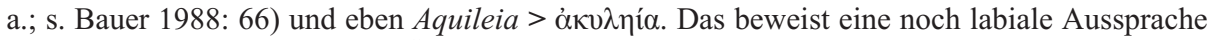
des griechischen $v$ zur Zeit der Übernahme dieser lateinischen lexikalischen Einheiten (dies sei als Ergänzung zu Karvounis 2008: 78-80 angemerkt). Zu lat. qui > gr. *[kü] s. Muljačić (2005:

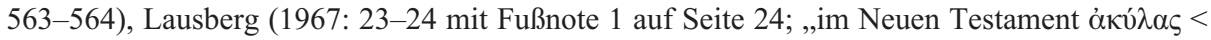

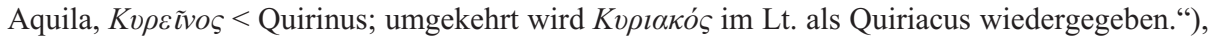
Lausberg (1967: 64), Lausberg (1969: 163).

30 Dass hier an die Stelle des zu erwartenden geschlossenen $\bar{e}$ ein offenes è getreten ist, erklärt Skok (1921-22: 30-31, 151) durch Einfluss der gelehrten kirchenlateinischen Aussprache. Ramovš (1921-1922: 33-34) nimmt darauf kritisch Bezug. Könnte es sich hier nicht auch um eine den Öffnungsgrad betreffende Dissimilation innerhalb des Diphthongs $(\bar{e} j>\bar{e} j)$ handeln? 
$37 * \beta \bar{y} \cdot d b n b>38 * \beta \overline{\underline{t}} \cdot d b n b>39 * \beta \underline{\bar{t}} \cdot d b n b>48 * \beta \underline{\bar{t}} \cdot d b n>55 * \beta \underline{\bar{t}} \cdot d a n>70$ čak. (mit hypothetischer Akzentuierung) $\dagger$ Vìdam, vgl. $z$ Vidma in der Istrischen Grenzziehung. Sloven. Videm, Gen. Vidma, dt. Weiden. Siehe Kranzmayer (1956: 43 Fußnote 17), Skok (1973: 586), Šimunović (1976: 12), Katičić (1980: 29), Šekli (2009: 125, 126), Snoj (2009: 454). Das auslautende $-m$ in den slavischen Namenlautungen geht auf eine hyperkorrekte Subtraktion des dialektalen Lautwandels $-m>-n$ zurück; ${ }^{31}$ anders Šekli (2009: 123 Fußnote 21).

\section{Koroška}

Substratidg. *Karantjā (vgl. Anreiter 2001: 46-47) > ursl. *Karantjāं (ohne Entlehnungs-Akut in der betonten Silbe) 'Kärnten'. Nach Markos Snojs brieflicher Mitteilung ist für diese unbelegte Basis vom Akzentparadigma $\mathrm{b}$ auszugehen, um die Akzentuierungen der Ableitungen neuštokavisch kòruški, sloven. koróški (tonemisch korộški) 'kärntnerisch', substantiviert Koróška, Koróško 'Kärnten', und Korošíca $(<*$ Koroščíca) 'Kärntnerin' erklären zu können. Vgl. auch Snoj (2009: 201).

Das resianische Koroten, Gen. Korotona 'Kärnten', dem, wie er mitteilt, schriftsprachliches Korotàn, Gen. Korotána entsprechen würde, leitet Marko Snoj von lat. Carantāni 'die Kärntner' her, das im Urslavischen Akut auf der betonten dritten Silbe bekommen habe. Im Resianischen sei, so Marko Snoj, dieser Plural auf dieselbe Weise singularisiert worden wie die Toponyme Rakičan aus Rakičane 'die bei den Weiden Wohnenden' (Prekmurje), maked. Rakištan und ukr. Poljan. In meiner Notation wäre also ursl. *Karant $\underline{\underline{a}} n e^{32}$ anzuschreiben (wohl ist hier das lateinische - $\underline{a} n i$ mit dem urslavischen Bewohnernamensuffix ${ }^{*}$-ja $\bar{n}$ ne volksetymologisch assoziiert worden).

\section{Zilja}

Substratsprachliches *Gīla (davon dt. Gail, 1090 Gila) $>$ ursl. *Gī ${ }^{*} j \bar{a}{ }^{*}$ (mit Entlehnungs-Akut) > 71635 u. a. sloven. Zílja. Siehe Holzer (1996b: 85, 2008: 75 § 1.5), Snoj (2009: 481). Nach Marko Snojs brieflicher Auskunft folgte der Name angesichts des Deminutivs Zíljica ‘Gailitz' dem Akzentparadigma a.

\section{Labot}

Alteurop. ${ }^{*}$ Albanta (vgl. uridg. ${ }^{*}$ alb $^{h} O-{ }^{\prime}$ 'wei $\left.{ }^{\prime 33}\right)>$ ursl. ${ }^{*}$ Al bantu (mit EntlehnungsAkut) $>10$ nachursl. *Lā bantu > sloven. Lábot und dt. dial. Lååfnt 'Lavant'. Snoj (2009: 222) führt sloven. Lábot, v Lábotu auf *Ölbotb zurück. Die slavische Initialbetonung lässt auf alteuropäische Initialbetonung schließen und zeigt, dass dieser Name nicht über das Lateinische bzw. Romanische, wo er pänultimalbetont gewesen sein musste, ins Slavische übernommen worden ist, sondern direkt aus dem vorrömischen Substrat (das das Romanische vielleicht sogar überlebt hatte).

31 Vgl. sloven. Píčem, ital. Pedena, dt. Piben, s. Kranzmayer (1956: 43 Fußnote 17).

32 Vgl. auch altruss. Xorutane, dessen $x$ auf bairische Vermittlung weist, s. Vasmer (1980: 266).

33 Zur Bedeutung von 'weiß' und 'schwarz' in Flussnamen s. Holzer (2008: 9-30). 


\section{Labe}

Alteurop. * $\underline{\text { Albis }}$ (so lat. belegt; gr. 'A $\lambda \beta 1$; ; vgl. uridg. *alb ${ }^{h} \mathrm{o}$ - 'weiß') > ursl. * $\underline{A l}$ 'bi (mit Entlehnungs-Akut) $>10$ u. a. tsch. Labě> Labe 'Elbe'. Ohne urslavischen Akut würde der Name im Tschechischen **Lobe lauten. Sloven. Lába ist aus dem Tschechischen entlehnt. Vgl. Snoj (2009: 521).

\section{Řezno}

Wohl aus dem Keltischen (s. Der Kleine Pauly IV 1366): Lat. Regina (Tabula Peutingeriana und Itinerarium Antoninianum 250), castra Regina (Notitia dignitatum XXXV 17; $\check{\imath}$ nach Der Kleine Pauly IV 1366), daraus ursl. *Regina $>7162325 *$ Redzbno $>3548$ u. a. tsch. Řezno 'Regensburg'.

Zur Regensburger Mission in Böhmen zu Beginn des 9. Jahrhunderts und zur Nachricht der Annales Fuldenses, nach der Ludwig der Deutsche am 13. Jänner 845 vermutlich in Regensburg vierzehn Fürsten aus Böhmen taufen ließ, siehe Nechutová (2007: 32).

\section{Vlah}

Ursl. *wal 'xu (mit Entlehnungs-Akut) 'Welscher' > (alt)russisch volox, kroatisch und serbisch 6202539434870 vläh 'Romane', sloven. (v)làh 'Italiener'. Nach der communis opinio aus dem Germanischen übernommen, von dem er seinerseits aus dem Keltischen (vgl. Caesar, De bello gallico VI 24: Volcae) entlehnt worden ist, bevor im Germanischen $k$ zu (c) $h$, verschoben“ wurde, was um das 4. Jh. v. Chr. geschehen sein könnte. Das dem damaligen Germanischen fremde keltische $o$ wurde durch $a$ substituiert. Nach der germanischen Lautverschiebung, aber vor den verschiedenen slavischen Entwicklungen der interkonsonantischen Sequenz al (südslavisch 20) gelangte der Name aus dem Germanischen ins Slavische. Siehe Pronk-Tiethoff (2013: 98-99), Snoj (2009: 224 s. v. Láhinja), Holzer (im Druck).

\section{Luga}

Aus einer dem finn. Laukaa gegenüberstehenden Stufenwechselform: Ursl. * Law ${ }^{\circ} g \bar{a}{ }^{*}$ (mit Entlehnungs-Akut) $>317$ russ. Luga. Ein westlich von St. Petersburg in den Finnischen Meerbusen mündender Fluss, der auf Finnisch Laukaa(njoki) heißt, was sich zu estn. laugas 'Sumpf, Morast' stellt. Siehe Vasmer (1971: 304, 1979: 65), Kiparsky (1948: 46), Strumiński (1996: 152), Holzer (2001: 39-40, 2008: 226), Kallio (2006: 160).

\section{Ladoga}

Entlehnung eines nicht näher identifizierbaren finnischen Wortes (nach Strumiński vermittelt durch anord. *Aldaga) ist ursl. * $\underline{\text { Al }} \cdot{ }^{*} \operatorname{dag} \bar{a}{ }^{*}$ (mit Entlehnungs-Akut) $>1023$ u. a. russ. Ladoga. Altisländisch Aldeigja (ca. 1010 belegt) und (späteres) Aldejgjuborg sowie mnd. Aldagen $(1268,1269)$ verbürgen, dass der Name ursprünglich mit $A l$ - anlautete. Siehe Vasmer (1979: 5), Strumiński (1996: 89-92, 234-235), Sitzmann (2003: 37-39).

Ladoga entstand als Siedlung um die Mitte des 8. Jh. (s. Janin 1986: 210-211, Goehrke 1992: 31, 34). Es gehörte zu den ,,an den großen Stromwegen entstehenden 
ältesten Handels- und Herrschaftszentren“, wo finnische, baltische, ostslavische und skandinavische Bevölkerungselemente zusammentrafen (s. Goehrke 1992: 35).

\section{Ižora}

Eine dem finn. Inkeri gegenüberstehende Stufenwechselform (nach Strumiński InGerä-) wurde vor der Ersten Palatalisierung und somit auch vor dem Wirken des Dyboschen Gesetzes entlehnt: Vorursl. * ${ }^{*} \bar{g}$ gerā ${ }^{*}>$ ursl. ${ }^{*} \underline{I}$ džer $\bar{a}{ }^{*}$ (ohne Entlehnungs-Akut) $>18$ altruss. Ižera > russ. Ižora. Linker Nebenfluss der Neva, das Land an diesem Fluss heißt schwedisch Ingermanland und mnd. Engeren. Siehe Strumiński (1996: 150-152), Holzer (2008: 223).

\section{Don}

Ursl. *Danu (mit bei Entlehnungen ungewöhnlicher Betonungslosigkeit, daher wohl aus einem früh entlehnten vorursl. *Danas, das - wohl über das Akzentparadigma D - der Regel entsprechend zum Akzentparadigma $\mathrm{C}$ übergewechselt ist, vgl. vorursl. *zambas $>$

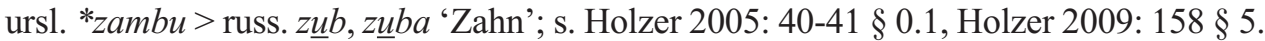
und $170 \S 12.3$, Holzer 2011b: 5 Fußnote 19)> 23253048 russ. Donn, Gen. Dona; ukr. Dịn, Gen. Donu. Davon das Deminutivum ursl. *Daniku > russ. Donęc. Nach Vasmer (1976: 362) aus dem Iranischen. Zum vielleicht kimmerisch vermittelten griechischen Namen Tóvaïs (Herodot, Strabo) s. Holzer (1989: 190-191).

\section{Dnestr}

Ursl. *Dunēstru oder *Dunajstru $>11242537$ u. a. aruss. Dъněstrъ $>48$ u. a. russ. Dnesstr, Gen. Dnestra , also entlehnt vor dem Wirken des Dyboschen Gesetzes (11). Abweichend akzentuiert ist das bei Konstantin Porphyrogennetos belegte $\Delta \alpha ́ v \alpha \sigma \tau \rho ı \varsigma$ (s. Moravcsik 1949: 291). Wohl iranisch. Vgl. noch lat. Danastrus (Jordanes), arab. Dānast. Vgl. Vasmer (1971: 159, 1976: 355).

\section{Dnepr}

Ursl. *Dunēpru oder *Dunajpru $>11242537$ u. a. aruss. Dъněprъ $>48$ u. a. russ. Dnẹpr, Gen. Dnepra , also entlehnt vor dem Wirken des Dyboschen Gesetzes (11). Abweichend akzentuiert ist das bei Konstantin Porphyrogennetos belegte $\Delta \alpha ́ v \alpha \pi \rho ı \varsigma$ (s. Moravcsik 1949: 291). Wohl iranisch. Vgl. noch lat. Danaper (Jordanes). Vgl. Vasmer (1971: 161, 1976: 354-355).

\section{Literatur}

ANREITER, Peter (1997) Breonen, Genaunen und Fokunaten. Vorrömisches Namengut in den Tiroler Alpen. Budapest: Archaeolingua Foundation. (Archaeolingua Series Minor = Innsbrucker Beiträge zur Kulturwissenschaft, Sonderheft 99.)

ANREITER, Peter (2001) Die vorrömischen Namen Pannoniens. Budapest: Archaeolingua Foundation. (Archaeolingua Series Minor, 16.) 
ARj I-XXIII: Rječnik hrvatskoga ili srpskoga jezika. I-XXIII. Zagreb: Jugoslavenska akademija znanosti i umjetnosti, 1881-1976.

BAUER, Walter ( $\left.{ }^{6} 1988\right)$ Griechisch-deutsches Wörterbuch zu den Schriften des Neuen Testaments und der frühchristlichen Literatur. 6., völlig neu bearbeitete Auflage, herausgegeben von Kurt Aland und Barbara Aland. Berlin/New York: Walter de Gruyter.

DER KLEINE PAULY IV: Der Kleine Pauly. Lexikon der Antike. Auf der Grundlage von Pauly's Realencyclopädie der classischen Altertumswissenschaft unter Mitwirkung zahlreicher Fachgelehrter bearbeitet und herausgegeben von Konrat Ziegler und Walther Sontheimer. Band 4 Nasidius - Scaurus. München: Deutscher Taschenbuch Verlag, 1979.

GEORGES, Karl Ernst (1861) Lateinisch-Deutsches Handwörterbuch aus den Quellen zusammengetragen und mit besonderer Bezugnahme auf Synonymik und Antiquitäten mit Berücksichtigung der besten Hülfsmittel ausgearbeitet von Dr. Karl Ernst Georges. Fünfte, dem heutigen Standpunkte der lateinischen Sprachwissenschaft gemäß umgestaltete Auflage. I. Leipzig: Hahn'sche Verlags-Buchhandlung.

GEORGES, Karl Ernst (1862) Lateinisch-Deutsches Handwörterbuch aus den Quellen zusammengetragen und mit besonderer Bezugnahme auf Synonymik und Antiquitäten mit Berücksichtigung der besten Hülfsmittel ausgearbeitet von Dr. Karl Ernst Georges. Fünfte, dem heutigen Standpunkte der lateinischen Sprachwissenschaft gemäß umgestaltete Auflage. II. Leipzig: Hahn'sche Verlags-Buchhandlung.

GOEHRKE, Carsten (1992), Frühzeit des Ostslaventums. Unter Mitwirkung von Ursel Kälin. Darmstadt: Wissenschaftliche Buchgesellschaft.

HER: Hrvatski enciklopedijski rječnik. Zagreb: Novi Liber, 2003.

HOLZER, Georg (1989) Entlehnungen aus einer bisher unbekannten indogermanischen Sprache im Urslavischen und Urbaltischen. Wien: Verlag der Österreichischen Akademie der Wissenschaften. (Sitzungsberichte der Philosophisch-Historischen Klasse der Österreichischen Akademie der Wissenschaften, 521.)

HOLZER, Georg (1996a) Das Erschließen unbelegter Sprachen. Zu den theoretischen Grundlagen der genetischen Linguistik. Frankfurt am Main usw.: Peter Lang. (Schriften über Sprachen und Texte, 1.)

HOLZER, Georg (1996b) „Zu Lautgeschichte und Dialekten des mittelalterlichen Slavischen in Österreich." Wiener Slavistisches Jahrbuch 42, 81-110.

HOLZER, Georg (1998) „Urslavisch und Baltisch.“ Wiener Slavistisches Jahrbuch 44, 27-56.

HOLZER, Georg (2001) „Zur Lautgeschichte des baltisch-slavischen Areals.“ Wiener Slavistisches Jahrbuch 47, 33-50.

HOLZER, Georg (2005) ,Zur relativen Datierung prosodischer Prozesse im Gemeinslavischen und frühen Kroatischen." Wiener Slavistisches Jahrbuch 51, 31-71.

HOLZER, Georg (2006) „Die Geschichte des Slavischen der Stadt Saloniki bis zum Jahr 863.“ In: J. Reinhart (Hrsg.), Slavica mediaevalia in memoriam Francisci Venceslai Mareš. (Schriften über Sprachen und Texte, 8.) Frankfurt am Main u. a.: Peter Lang, 29-67. 
HOLZER, Georg (2007) Historische Grammatik des Kroatischen. Einleitung und Lautgeschichte der Standardsprache. Frankfurt am Main u. a.: Peter Lang. (Schriften über Sprachen und Texte, 9.)

HOLZER, Georg (2008) Namenkundliche Aufsätze. Wien: Praesens Verlag. (Innsbrucker Beiträge zur Onomastik, 4.)

HOLZER, Georg (2009) „Urslavische Prosodie.“ Wiener Slavistisches Jahrbuch 55, 151-178. HOLZER, Georg (2011a) „Zur Akzentuierung romanisch vermittelter Gräzismen im Kroatischen.“ In: E. Stadnik-Holzer (Hrsg.), Baltische und slavische Prosodie. International Workshop on Balto-Slavic Accentology IV (Scheibbs, 2.-4. Juli 2008). Scheibbser Internationale Sprachhistorische Tage I. (Schriften über Sprachen und Texte, 11.) Frankfurt am Main u. a.: Peter Lang, 81-88.

HOLZER, Georg (2011b) Glasovni razvoj hrvatskoga jezika. Zagreb: Institut za hrvatski jezik i jezikoslovlje. [Übersetzung einer ergänzten und verbesserten Version von Holzer 2007.]

HOLZER, Georg (2014) „Rekurrente Unregelmäßigkeiten in alten südslavischen Romanismen.“ In: G. Rampl/K. Zipser/M. Kienpointner (Hrsg.), In Fontibus Veritas. Festschrift für Peter Anreiter zum 60. Geburtstag. Innsbruck: innsbruck university press, 199-211.

HOLZER, Georg (im Druck) „Der Walchen-Name im frühmittelalterlichen Slavischen.“ In: W. Pohl (Hrsg.), Romanen und ihre Fremdbezeichnungen im Mittelalter: Walchen, Vlachen, Waliser. Wien: Verlag der Österreichischen Akademie der Wissenschaften.

JANIN, Valentin Lavrent'evič (1986) „Ladoga und Nowgorod.“ In: J. Herrmann (Hrsg.), Welt der Slawen. Geschichte, Gesellschaft, Kultur. München: Verlag C. H. Beck, 210-214.

KALLIO, Petri (2006) „On the Earliest Loanwords in Finnic.“ In: J. Nuorluoto (Hrsg.), The Slavicization of the Russian North. Mechanisms and Chronology. (Slavica Helsingiensia, 27). Helsinki: Department of Slavonic and Baltic Languages and Literatures, $154-166$.

KARVOUNIS, Christos (2008) Aussprache und Phonologie im Altgriechischen. Darmstadt: Wissenschaftliche Buchgesellschaft.

KATIČIĆ, Radoslav (1980) „Slavica Foroiuliensia.“ Wiener Slavistisches Jahrbuch 26, 28-32.

KIPARKSY, Valentin (1948) „Chronologie des relations slavobaltiques et slavofinnoises.“ Revue des Études slaves 24, 29-47.

KLOTZ, Reinhold (1874) Handwörterbuch der lateinischen Sprache. I-II. 5. Auflage. Braunschweig: George Westermann.

KRANZMAYER, Eberhard (1956) Ortsnamenbuch von Kärnten. I. Teil: Die Siedlungsgeschichte Kärntens von der Urzeit bis zur Gegenwart im Spiegel der Namen. Klagenfurt: Verlag des Geschichtsvereines für Kärnten.

LAUSBERG, Heinrich (1967) Romanische Sprachwissenschaft II: Konsonantismus. 2. Aufl. Berlin: Walter de Gruyter \& Co.

LAUSBERG, Heinrich (1969) Romanische Sprachwissenschaft I: Einleitung und Vokalismus. 3. Aufl. Berlin: Walter de Gruyter \& Co. 
MORAVCSIK, Gy. (Hrsg.) (1949) Constantine Prophyrogenitus De Administrando Imperio. Greek text edited by Gy. Moravcsik. English translation by R. J. H. Jenkins. Budapest: Pázmány Péter Tudományegyetemi Görög Filológiai Intézet.

MULJAČIĆ, Žarko (2005) „Il vocalismo del latino di Krk/Veglia (e del veglioto primigenio) sotto l'influsso del greco (e del croato)." In: P. S. Ureland (Hrsg.), Integration of European Language Research (Studies in Eurolinguistics, 2. = Proceedings of the 3rd Eurolinguistics Symposium "A Typology of Language Contacts Around the Baltic and Adriatic Sea", Zadar (Croatia), 19-22 September 2002). Berlin: Logos Verlag, 563-569.

NECHUTOVÁ, Jana (2007) Die lateinische Literatur des Mittelalters in Böhmen. Köln: Böhlau.

POPOVIĆ, Ivan (1960) Geschichte der serbokroatischen Sprache. Wiesbaden: Otto Harrassowitz.

PRAVOPIS 1960: Pravopis srpskohrvatskoga književnog jezika sa pravopisnim rečnikom. Novi Sad/Zagreb: Matica srpska - Matica hrvatska.

PRONK-TIETHOFF, Saskia (2013) The Germanic loanwords in Proto-Slavic. Amsterdam/New York: Rodopi. (Leiden Studies in Indo-European, 20.)

RAMOVŠ, Franc (1921-1922) „Slov. Celje.“ Časopis za slovenski jezik, književnost in zgodovino III, 33-34.

RAMOVŠ, Franc (1921-1922) „Slov. Kobarid-furl. Ćavorèd - ital. Caporetto - nem. Karfreit." Časopis za slovenski jezik, književnost in zgodovino III, 60-62.

RISTIĆ, Svetomir/Jovan KANGRGA (1928) Rečnik srpskohrvatskog i nemačkog jezika. Drugi deo srpskohrvatsko-nemački. Beograd: Izdavačka knjižarnica Rajkovića i Ćukovića.

RIX, Helmut (1976) Historische Grammatik des Griechischen. Laut- und Formenlehre. Darmstadt: Wissenschaftliche Buchgesellschaft.

SCHRAMM, Gottfried (1981) Erobererund Eingesessene. Geographische Lehnnahmen als Zeugen der Geschichte Südosteuropas im ersten Jahrtausend n. Chr. Stuttgart: Anton Hersemann Verlag.

ŠEKLI, Matej (2009) „On Romance-Alpo-Slavic substitutional accentology: the case of pre-Slavic masculine substrate place names in Slovene." In: Th. Olander/J. H. Larsson (Hrsg.), Stressing the past. Papers on Baltic and Slavic accentology. Amsterdam/New York: Rodopi, 145-160.

ŠIMUNOVIĆ, Petar (1976) „Toponimija Istarskoga razvoda. U povodu sedamstogodišnjeg jubileja 1275. - 1975.“ Onomastica Jugoslavica 6, 3-34.

ŠIMUNOVIĆ, Petar (1986) Istočnojadranska toponimija. Split: Logos.

SITZMANN, Alexander (2003) Nordgermanisch-ostslavische Sprachkontakte in der Kiever Rus' bis zum Tode Jaroslavs des Weisen. Wien: Edition Praesens.

SKOK, Petar (1921-22) „Oglej i Celje.“ Časopis za slovenski jezik, književnost in zgodovino III, 24-32 und 151.

SKOK, Petar (1950) Slavenstvo i romanstvo na jadranskim otocima. Toponomastička ispitivanja 1. Zagreb: Jadranski institut Jugoslavenske akademije znanosti i umjetnosti. SKOK, Petar (1953) „Uslovi života glagoljice.“ Slovo 3, 50-63.

SKOK, Petar (1972) Etimologijski rječnik hrvatskoga ili srpskoga jezika II. Zagreb: Jugoslavenska akademija znanosti i umjetnosti. 
SKOK, Petar (1973) Etimologijski rječnik hrvatskoga ili srpskoga jezika III. Zagreb: Jugoslavenska akademija znanosti i umjetnosti.

SNOJ, Marko (2009) Etimološki slovar slovenskih zemljepisnih imen. Ljubljana: Modrijan - Založba ZRC.

STRUMIŃSKI, Bohdan (1996) Linguistic Interrelations in Early Rus'. Northmen, Finns, and East Slavs (Ninth to Eleventh Centuries). Roma/Edmonton/Toronto: Canadian Institute of Ukrainian Studies Press. (Collana di filologia e letterature slave, II.)

VASMER, Max (1971) Schriften zur slavischen Altertumskunde und Namenkunde herausgegeben von Herbert Bräuer. Berlin/Wiesbaden: Otto Harrassowitz. [I. Band bis Seite 534, II. Band (nach I-VIII) ab Seite 537.]

VASMER, Max (1976) Russisches etymologisches Wörterbuch I. Heidelberg: Carl Winter - Universitätsverlag.

VASMER, Max (1979) Russisches etymologisches Wörterbuch II. Heidelberg: Carl Winter - Universitätsverlag.

VASMER, Max (1980) Russisches etymologisches Wörterbuch III. Heidelberg: Carl Winter - Universitätsverlag.

\section{Resümee \\ LAUT- UND AKZENTGESCHICHTLICHES ZU EINIGEN AUS SUBSTRATEN UND NACHBARSPRACHEN INS SLAVISCHE ENTLEHNTEN NAMEN}

In diesem Beitrag wird die Laut- und Akzentgeschichte einiger geographischer Namen untersucht, die das Slavische der Expansionszeit oder kurz danach von seinen Substratoder neuen Nachbarsprachen übernommen hat. Die ausgewählten Namen stammen aus dem Griechischen, Lateinischen bzw. Romanischen, „Alteuropäischen“, Thrakischen und anderen indogermanischen Substratsprachen wie auch aus dem Deutschen und anderen germanischen Sprachen, aus dem Finnischen und dem Iranischen. Im Einzelnen werden folgende Namen behandelt: *Silicānum > Solkan, *Silicêetum > Sužid,

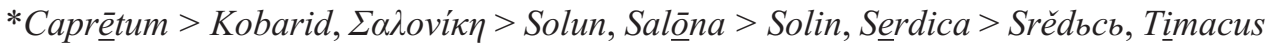
$>$ Timok, Sancta Maria $>$ Sutomore, Sancta Marīa $>$ Sutomorje, Longaticum $>$ Logatec,

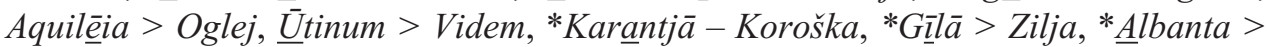
Labot, Albis $>$ Labe, Regina $>$ Řezno, *wal xu > Vlah, Laukaa - Luga, ursl. * ${ }^{*}$ l 'dagā $>$ Ladoga, Inkeri - Ižora, ursl. *Danu > Don, *Dunēstru oder *Dunajstru > Dnestr, *Dunēpru oder *Dunajpru > Dnepr.

Schlüsselwörter: Namen, Entlehnung, Substrate, Akzent, Lautgesetze 


\section{Abstract \\ REMARKS ON THE HISTORY OF SOUNDS AND ACCENTS \\ OF SLAVIC NAMES DERIVING FROM SUBSTRATES AND \\ NEIGHBOURING LANGUAGES}

This paper examines the history of sounds and accents of a number of place names that have been borrowed by Slavic languages from substrate or neighbouring languages around the time of the Slavic expansion. The selected names originate from Greek, Latin and Romance, "Ancient European", Thracian and other Indo-European substrate languages as well as from German and other Germanic dialects, Finnish and Iranian. The following names are examined in detail: *Silicānnum $>$ Solkan, ${ }^{*}$ Silicêetum $>$ Sužid,

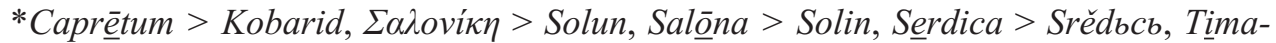

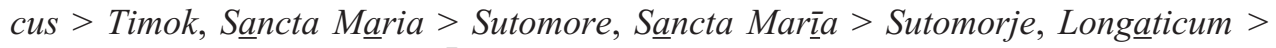

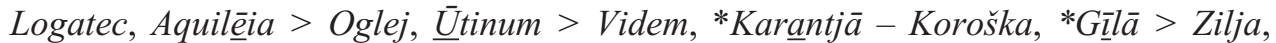
* Albanta > Labot, Albis > Labe, Reginna > Řezno, *wal xu > Vlah, Laukaa - Luga, Proto-Sl. * ${ }^{*}$ l ${ }^{*} \operatorname{dag} \bar{a}{ }^{*}>$ Ladoga, Inkeri - Ižora, Proto-Sl. *Danu > Don, *Dunēstru or *Dunajstru $>$ Dnestr, *Dunępru or *Dunajpru $>$ Dnepr.

Keywords: names, loans, substrates, accent, sound laws

\section{Povzetek}

\section{K ZGODOVINI GLASOV IN NAGLASOV V SLOVANSKIH LASTNIH IMENIH, PREVZETIH IZ SUBSTRATNIH IN SOSEDNJIH JEZIKOV}

Članek se ukvarja z zgodovino glasov in naglasov $\mathrm{v}$ številnih zemljepisnih imenih, ki so bili v slovanske jezike prevzeti iz substratnih ali iz sosednjih jezikov v času slovanske širitve. Izbrana imena izhajajo iz grščine, latinščine oz. romanskih različic, nato iz »stare indoevropščine«, trakijščine in drugih indoevropskih substratnih jezikov pa tudi iz nemščine in drugih germanskih narečij, finščine in iranščine. V razpravi so natančno proučena naslednja imena: *Silicānum $>$ Solkan, ${ }^{*}$ Silicẹtum $>$ Sužid, ${ }^{*}$ Caprētum $>$

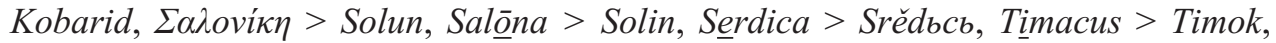
Sancta Maria $>$ Sutomore, Sancta Marīa $>$ Sutomorje, Longaticum $>$ Logatec, Aquilēia

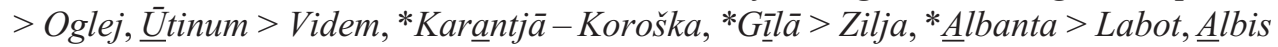
$>$ Labe, Regina > Řezno, *wal 'xu > Vlah, Laukaa - Luga, psl. * ${ }^{*}$ Al 'dagā $>$ Ladoga,

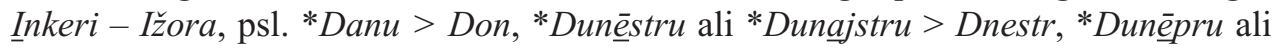
*Dunajpru $>$ Dnepr.

Ključne besede: lastna imena, izposojenke, substrat, naglas, glasovni zakoni 